Research in Astronomy and Astrophysics manuscript no.

(LTEX: ms1391.tex; printed on October 17, 2018; 18:25)

\title{
A two-step energy injection explanation for the rebrightenings of the multi-band afterglow of GRB 081029 *
}

\author{
Yong-Bo $\mathrm{Yu}^{1,2}$ and Yong-Feng Huang ${ }^{1,2}$ \\ ${ }^{1}$ Department of Astronomy, Nanjing University, Nanjing 210093, China; hyf@nju.edu.cn \\ 2 Key Laboratory of Modern Astronomy and Astrophysics (Nanjing University), Ministry of Education, \\ Nanjing 210093, China
}

\begin{abstract}
The afterglow of GRB 081029 showed unusual behavior, with a significant rebrightening being observed at optical wavelength at about $3000 \mathrm{~s}$ after the burst. One possible explanation is that the rebrightening is resulted from energy injection. Here, we present a detailed numerical study of the energy injection process and interpret the X-ray and optical afterglow light curves of GRB 081029. In our model, we have assumed two periods of energy injection, each with a constant injection power. One injection starts at $2.8 \times 10^{3} \mathrm{~s}$ and lasts for about $2500 \mathrm{~s}$, with a power of $7.0 \times 10^{47} \mathrm{erg} \mathrm{s}^{-1}$. This energy injection is mainly engaged to account for the rapid rebrightening at about $3000 \mathrm{~s}$. The other injection starts at $8.0 \times 10^{3} \mathrm{~s}$ and lasts for about $5000 \mathrm{~s}$. The injection power is $3.5 \times 10^{47} \mathrm{erg} \mathrm{s}^{-1}$. This energy injection can help to explain the slight rebrightening at about $10000 \mathrm{~s}$. It is shown that the observed optical afterglow, especially the marked rebrightening at about $3000 \mathrm{~s}$, can be well reproduced. In X-ray band, the predicted amplitude of the rebrightening is much shallower, which is also consistent with the observed X-ray afterglow light curve. It is argued that the two periods of energy injection can be produced by the falling of clumpy materials onto the central compact object of the burster, which leads to an enhancement of accretion and gives birth to a strong outflow temporarily.
\end{abstract}

Key words: gamma rays: bursts - ISM: jets and outflows - individual: GRB 081029

\section{INTRODUCTION}

Gamma-ray bursts (GRBs) are bright flashes of gamma-rays coming from random directions in the sky at random times (for recent reviews, see Zhang 2007, Gehrels et al. 2009). The fireball model is very successful and popular in view of the fact that it can well explain the main features of GRB afterglows (Rees \& Méseáros 1994; Piran 1999; Zhang 2007), which are generally believed to arise from the interaction of the fireball with the surrounding interstellar medium (ISM) (Méseáros \& Rees 1997a; Piran 2000; Méseáros 
2002). After the amazing coincidence of GRB 980425 and SN 1998bw (Galama et al. 1998), more and more observational facts have been accumalated (for recent review, see Bersier 2012), indicating that long GRBs are associated with Type Ic supernovae. Based on these observations, it is believed that long GRBs should be due to the collapse of massive stars (Woosley 1993; Paczyński 1998; MacFadyen \& Woosley 1999). At the same time, it is also widely accepted that short GRBs could be connected with the coalescence of two compact objects (Eichler et al. 1989; Narayan et al. 1992; Gehrels et al. 2005; Nakar 2007).

With the advance of observational techniques, especially after the launch of the Swift satellite, many unexpected behaviors are observed in GRB afterglows, such as quick or high amplitude rebrightenings in optical band, and strong or multiple flares at X-ray wavelength (for recent review, see Zhang 2007). GRB 081029 is one of the interesting events, which has a remarkable rebrightening in its optical afterglow light curve. Other examples include GRB 060206 (Wózniak et al. 2006) and GRB 970508 (Sokolov et al. 1998). These rebrightenings are obviously inconsistent with the simple form of power-law decay as predicted by the standard fireball model with synchrotron emission coming from the forward shock of ejecta ploughing into an external medium (Rhoads 1999; Sari et al. 1999). Many different mechanisms have been proposed to explain the rebrightenings, including the density jump model (Lazzati et al. 2002; Dai \& Wu 2003; Tam et al. 2005), the energy injection model (Dai \& Lu 1998; Rees \& Méseáros 1998; Huang et al. 2006), the twocomponent jet model (Huang et al. 2004; Liu et al. 2008), and the microphysics variation mechanism (Kong et al. 2010), etc. However, in the more detailed numerical simulations, Huang et al. (2007) argued that the density jump model is not an ideal mechanism to produce the rebrightenings in optical afterglows. Holland et al. (2012) also ruled out the possibility that the extremely steep rebrightening of the optical afterglow of GRB 081029 is resulted from the density structure in the surrounding environment, due to the fact that it was unable to reproduce the magnitude of the increase in luminosity. Interestingly, Holland et al. (2011) reproduced some of the X-ray and optical/infrared rebrightenings reasonably well with the two-component jet model. In this model, the early afterglow emission is produced by the narrow, fast component while the wider, slower component dominates the afterglow after about $3000 \mathrm{~s}$. But their calculations still failed to reproduce the rapid rise as seen in the UVOT data.

Energy injection from late and slow shells seems to be a natural interpretation for the rebrightening of many optical afterglows. Especially, GRB 970508 exhibited a late-time flare similar to what is expected from colliding shells (Sokolov et al. 1998). In this study, we will use a two-step energy injection mechanism to explain the observed unusual X-ray and optical afterglow light curves of GRB 081029. In our calculation, we only consider the synchrotron emission, which is the dominant radiation mechanism that takes place in the afterglow stage, although inverse Compton scattering may also play a role in some cases (Wei \& Lu 2000; Sari \& Esin 2001). The outline of our paper is as follows. The observational facts are presented in Section 2. The two-step energy injection model, including the dynamics and the radiation process, are described in Section 3. In Section 4, we calculate the overall dynamical evolution of the outflow numerically, and reproduce the unusual X-ray and optical afterglow light curves of GRB 081029. It is shown that the observed rebrightening in the optical band can be well reproduced. Finally, in Section 5, we summarize our results and give a brief discussion. We use an assumptive cosmology of $H_{0}=71 \mathrm{~km} \mathrm{~s}^{-1} \mathrm{Mpc}^{-1}, \Omega_{M}=$ 


\section{DATA}

At 01:43:56 UT on 2008 Oct 29, GRB 081029 triggered the Burst Alert Telescope (BAT) onboard the Swift satellite and was located at coordinates $R A(J 2000)=23^{h} 07^{m} 06^{s}, \operatorname{Dec}(J 2000)=-68^{\circ} 10^{\prime} 43.4^{\prime \prime}$ (Cummings et al. 2008). The peak flux of GRB 081029 measured by the BAT in the $15-150 \mathrm{keV}$ band was $(2.8 \pm 1.3) \times 10^{-8} \mathrm{erg} \mathrm{cm}^{-2} \mathrm{~s}^{-1}$ with the duration of $T_{90}=280 \pm 50 \mathrm{~s}$. The spectrum was best fit by a simple power law with a photon index of $\Gamma=1.5 \pm 0.2$ (Holland et al. 2011), and the redshift measured by the VLT/UVES and Gemini-South from several absorption features in the host galaxy of GRB 081029 was $z=3.8479$ ( Nardini et al. 2011). The luminosity distance between GRB 081029 and the earth is $3.5 \times 10^{7}$ kpc for a standard cosmology with $\Omega_{M}=0.27, \Omega_{\Lambda}=0.73$ and $H_{0}=71 \mathrm{~km} \mathrm{~s}^{-1} \mathrm{Mpc}^{-1}$. A number of ground-based telescopes performed follow-up observations, providing the multi-frequency light curves of the afterglow. GRB 081029 was an unusual event with an unusual optical light curve among the GRBs. One of the most remarkable features of this burst is that the optical light curve has a significant rebrightening at around $3000 \mathrm{~s}$.

\subsection{Optical Afterglow}

The optical afterglow of GRB 081029 was identified by ROTSE located at the H.E.S.S. site at Mt. Gamsberg, Namibia, $86 \mathrm{~s}$ after the burst. The REM telescope equipped with the ROSS optical spectrograph/imager and the REMIR near-infrared camera started observing the optical afterglow $154 \mathrm{~s}$ after the BAT trigger in the R, J, and H bands. GROND, mounted at the 2.2m MPI/ESO telescope at La Silla, started observing the field of GRB 081029 about 8 minutes after the trigger. A steep rise was observed in all seven available optical and NIR bands. The Swift/UVOT began observing the afterglow of GRB 081029 at $2689 \mathrm{~s}$ after the BAT trigger, and the afterglow was detected in the $v, b$, and white bands, which was consistent with the reported redshift of $\mathrm{z}=3.8479$ (Holland et al. 2012). The $\mathrm{R}$ band light curve shows many interesting features, and is very different from the optical afterglow of a typical GRB. Firstly, the initial light curve decayed in the normal way with the simple power-law extrapolation, but the afterglow rebrightened significantly and rapidly at about $3000 \mathrm{~s}$ after the trigger, interrupting the smooth early-time temporal evolution, which cannot be explained by using the standard afterglow model. The obtained light curve confirmed the rebrightening from $r^{\prime} \sim 18.6$ magnitude to a peak value of $r^{\prime} \sim 17.4$ magnitude, probably implying a sudden release of a large amount of energy at late times. Secondly, the optical afterglow light curve became a little flat at around 8000 s. Finally, the afterglow flattened again after about two days, suggesting either the presence of an underlying dim host galaxy or a further change in the optical decay index (Nardini et al. 2011).

\subsection{X-ray Afterglow}

Owing to observing constraints (Sakamoto et al. 2008), XRT and UVOT onboard the Swift satellite started to follow-up GRB 081029 about 45 minutes after the BAT trigger, but X-ray observations continued for approximately 10 days. The X-ray afterglow light curve shows a shallow initial decay followed by a rapid decay, but does not show strong evidence for a marked rebrightening as compared to the optical afterglow, 
it should be noted that there is some evidence for flaring between approximately $2500 \mathrm{~s}$ and $5000 \mathrm{~s}$. The X-ray light curve could be described by a broken power law $\left(f_{\nu}=t^{-\alpha}\right)$ and the best-fitting model has decay indices of $\alpha_{1}=0.56 \pm 0.03$ and $\alpha_{2}=2.56 \pm 0.09$, with a break time of $t_{b}=18230 \pm 346 \mathrm{~s}$ (Holland et al. 2011). The Swift/XRT spectrum can be fit by a single power law function $\left(f_{\nu}=\nu^{-\beta}\right)$ with an index of $\beta=$ $0.98 \pm 0.08$. There is no evidence for any evolution in the power law index at X-ray energies (Holland et al. 2012).

\section{MODEL}

In recent years, Eerten et al. (2010) developed a code for the dynamical evolution of GRB afterglows. Their calculations include some delicate ingredient and are relatively accurate. But their code is also relatively complicate. Here we will use the simple equations for beamed GRB ejecta developed by Huang et al (1998, 1999a, 1999b, 2000a, 2000b) to describe the dynamic and radiation process of the afterglows of GRB 081029. These equations are applicable to both radiative and adiabatic blastwaves, and are appropriate for both ultra-relativistic and non-relativistic stages (Huang et al. 1999a, 1999b, 2000a, 2000b, 2003). Most importantly, it takes the lateral expansion, the cooling of electrons, and the equal arrival time surface effect into consideration. The evolution of radius $(R)$, the swept-up mass $(m)$, the half opening angle $(\theta)$ and the Lorentz factor are described as:

$$
\begin{gathered}
\frac{d R}{d t}=\beta c \gamma\left(\gamma+\sqrt{\gamma^{2}-1}\right) \\
\frac{d m}{d R}=2 \pi R^{2}(1-\cos \theta) n m_{p} \\
\frac{d \theta}{d t}=\frac{c_{s}\left(\gamma+\sqrt{\gamma^{2}-1}\right)}{R} \\
\frac{d \gamma}{d m}=\frac{-\left(\gamma^{2}-1\right)}{M_{e j}+\epsilon m+2(1-\epsilon) \gamma m}
\end{gathered}
$$

where $\beta=\sqrt{\gamma^{2}-1} / \gamma, n$ is the number density of surrounding ISM, $m_{p}$ is the mass of the proton, $c_{s}$ is the co-moving sound speed, $R$ is the distance from the center in the burster's frame, $t$ is the observer's time, $M_{e j}$ is the initial ejecta mass, $m$ is the swept-up ISM mass, and $\epsilon$ is the radiative efficiency. A reasonable expression for $c_{s}$ is:

$$
c_{s}^{2}=\hat{\gamma}(\hat{\gamma}-1)(\gamma-1) \frac{1}{1+\hat{\gamma}(\gamma-1)} c^{2},
$$

where $\hat{\gamma} \approx(4 \gamma+1) /(3 \gamma)$ is the adiabatic index (Dai et al. 1999).

In the standard fireball model, as the blast wave sweeps up the surrounding medium, the shock accelerates electrons. The afterglow emission arises from synchrotron radiation of these shocked electrons due to their interaction with magnetic field. Considering the energy injection, the differential equations should be modified accordingly so that it can be applicable to our case. Due to strong magnetic field and rapid rotation, a new-born millisecond pulsar will lose its rotation energy through magnetic dipole radiation. Dai \& Lu (1998) have considered the energy injection from a new-born strongly magnetized millisecond pulsar at the center of GRB. They argued that the radiation power evolves with time as $L(t)=L_{0}(1+t / T)^{-2}$, where $L_{0}$ is the initial luminosity, $t$ is the time in the burster's rest frame, and $T$ is the spin-down timescale. Considering an adiabatic relativistic hot shell which receives the energy injection from the central engine 
$L(t) \propto t^{q}$, where $t$ is the intrinsic time of the central engine. They pointed out that usually $q=0$ during the injection phase in many cases. To explain the special behaviors of GRB 070610 in the observed X-ray and optical afterglow light curves, Kong \& Huang (2010) assumed that the energy injection power takes the form of $d E_{\text {inj }} / d t=Q t^{q}$ for $t_{\text {start }}<t<t_{\text {end }}$, where $\mathrm{Q}$ and $\mathrm{q}$ are constants, $t_{\text {start }}$ is the beginning time of the energy injection, and $t_{\text {end }}$ is the ending time of the energy injection. For some types of central engines, such as a black hole plus a long-lived debris torus system, the energy injection to the fireball may in principle continue for a time scale significantly longer than that of the gamma-ray emission (Zhang \& Méseáros 2001a). Taking into account all the energy injection forms as described above, and the extremely fast optical rebrightening of the afterglow of GRB 081029 at about $3000 \mathrm{~s}$ after the trigger time, here we take the same form of energy injection power as Kong \& Huang (2010). The differential equation for the evolution of the bulk Lorentz factor (i.e. Eq. (4) ) should then be changed to:

$$
\frac{d \gamma}{d t}=\frac{1}{M_{e j}+\epsilon m+2(1-\epsilon) \gamma m} \times\left(\frac{1}{c^{2}} \frac{d E_{i n j}}{d t}-\left(\gamma^{2}-1\right) \frac{d m}{d t}\right) .
$$

In the simplest case, $\mathrm{q}=0$.

\section{NUMERICAL RESULTS}

Paying special attention to the rebrightening of the afterglow of GRB 081029 in the optical band at around $3000 \mathrm{~s}$, we use the energy injection model to calculate the X-ray and optical afterglow light curves in detail, and compare the numerical results with the observations. As the fluence of GRB 081029 measured in the 15 - $350 \mathrm{keV}$ energy range by BAT is $2.1 \pm 0.2 \times 10^{-6} \mathrm{erg} \mathrm{cm}^{-2}$ (Nardini et al. 2011), the isotropic energy released in the rest-frame in the $15-350 \mathrm{keV}$ band is then $E_{0, \text { iso }}=3.1 \times 10^{53} \mathrm{erg}$. In our calculations, we will assume this value as the initial isotropic kinetic energy of the outflow. Other parameters are taking as following: the initial Lorentz factor of the blast wave $\gamma_{0}=70$, the ISM number density $n=2.0 \mathrm{~cm}^{-3}$, the power-law index of the energy distribution of electrons $p=2.4$, the luminosity distance of the source $D_{L}$ $=3.5 \times 10^{7} \mathrm{kpc}$, the electron energy fraction $\epsilon_{e}=0.04$, the magnetic energy fraction $\epsilon_{B}=0.004$, the initial half opening angle of the ejecta $\theta=0.04$ radian, and the observing angle $\theta_{o b s}=0$, where the observing angle is defined as the angle between the line of sight and the jet axis.

For the significant rebrightening at approximately 3000s after the trigger, we assume an energy luminosity with $Q=7.0 \times 10^{47} \mathrm{erg} \mathrm{s}^{-1}, q=0, t_{\text {start }}=2.8 \times 10^{3} \mathrm{~s}$, and $t_{\text {end }}=5.3 \times 10^{3} \mathrm{~s}$. This will lead to a total energy injection of $E_{i n j}=3.1 E_{0}$, where $E_{0}=(1-\cos \theta) E_{0, i s o}$ is the collimation-corrected energy. According to the analysises by Zhang \& Méseáros (2002), such an injected energy higher than that of the original kinetic energy of the outflow should be able to generate an obvious rebrightening in the afterglow lightcurve. To get the best fit to the observations of GRB 081029 in the optical band, another energy injection process is required, which gives birth to the observed flat stage occurring between about $8000 \mathrm{~s}$ and 13000s. The parameters corresponding to this second energy injection are: $Q=3.5 \times 10^{47} \mathrm{erg} \mathrm{s}^{-1}, q=0$, $t_{\text {start }}=8.0 \times 10^{3} \mathrm{~s}$, and $t_{\text {end }}=1.3 \times 10^{4} \mathrm{~s}$. Additionally, contribution from a host galaxy with the magnitude of $r^{\prime} \sim 25$ mag is assumed, which will account for the final flat stage of the optical afterglow.

Using the model and parameters described above, we can give a satisfactory fit to the observed X-ray 


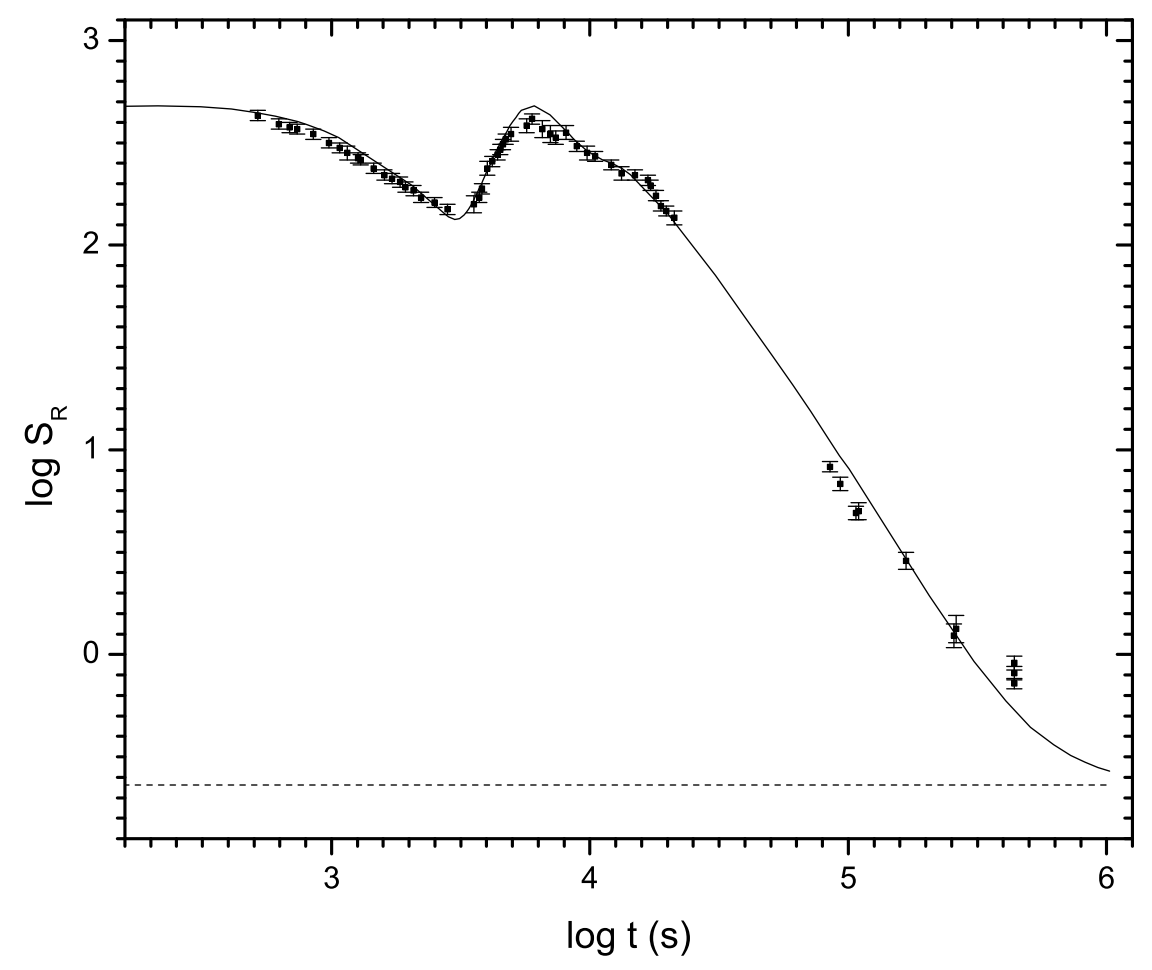

Fig. 1 Numerical fit to the optical afterglow (in units of $\mu \mathrm{Jy}$ ) of GRB 081029 by using the twostep energy injection model. The observational data are taken from Nardini et al (2011). The solid line is our theoretical optical afterglow light curve corrected for extinction. The dashed line is the contribution from a host galaxy with the magnitude of $25 \mathrm{mag}$.

from Nardini et al (2011). Also plotted are our calculated $R$ band flux densities $\left(S_{R}\right)$. We see that the observed optical afterglow light curve can be satisfactorily reproduced.

Figure 2 illustrates the observed X-ray light curve $\left(F_{X}\right)$ of GRB 081029 in the $0.3-10 \mathrm{keV}$ band. Observational data are taken from Nardini et al (2011). No rebrightening as significant as in the optical band could be identified. But Holland et al. (2011) argued that there could be some flares in the observed $\mathrm{X}$-ray light curve between approximately $2500 \mathrm{~s}$ and $5000 \mathrm{~s}$, and the time scale of the flares were $\Delta t / t<1$. However, the error bars of the observational data are generally large (as compared with optical data), so that no firm conclusion could be drawn. Also plotted in Figure 2 is our theoretical light curve by using the same energy injection model with the same parameters as in Figure 1. It is interesting to note that in X-ray band, the theoretical amplitude of the rebrightening due to energy injection is much smaller as compared with that at optical wavelength. Our numerical results are then actually well consistent with the observed X-ray light curve.

It should be noted that in our calculations, we do not consider the reverse shock emission component during the energy injection stage. Actually, depending on different types of central engines, the injected 


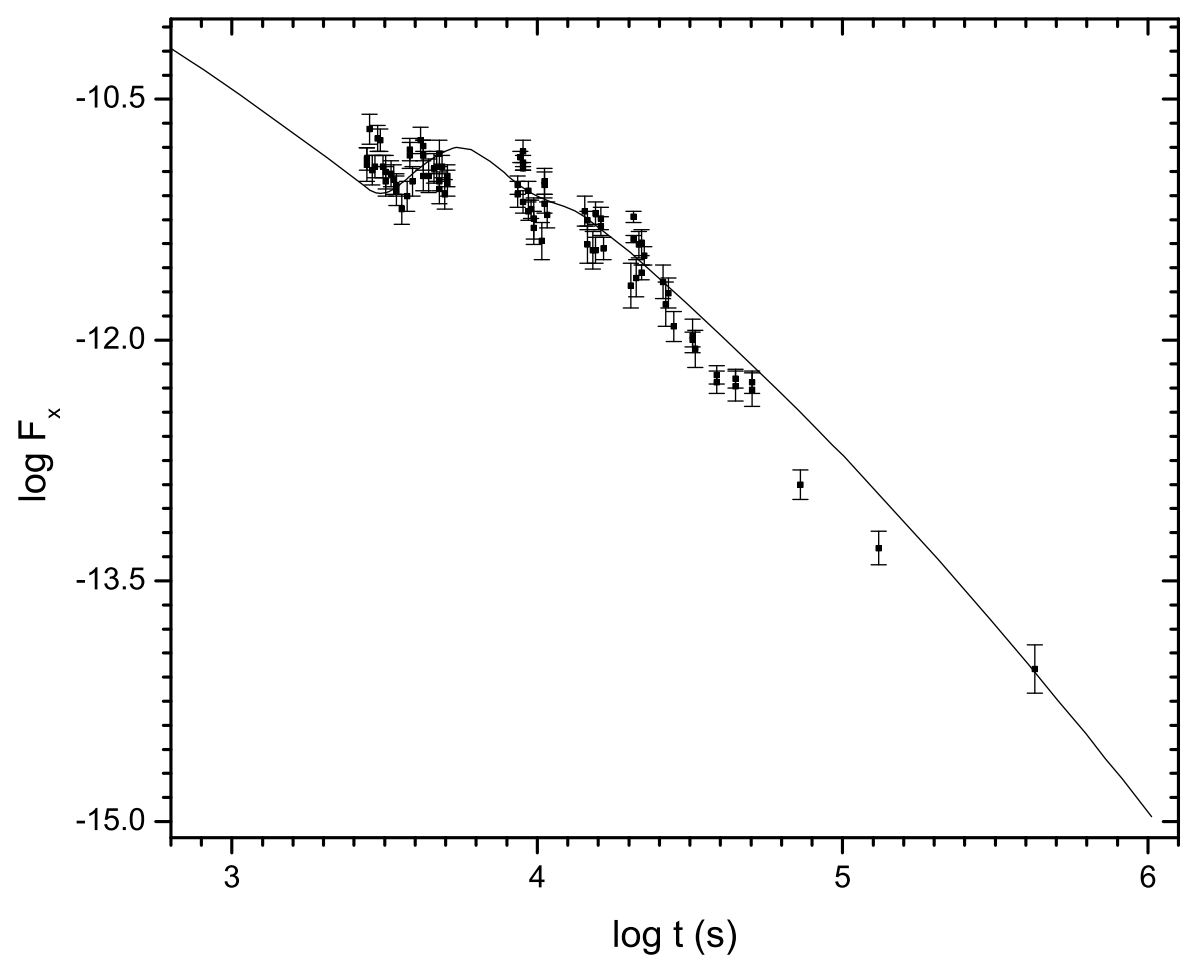

Fig. 2 Our numerical fit to the X-ray afterglow (in units of $\mathrm{erg}^{\mathrm{cm}}{ }^{-2} \mathrm{~s}^{-1}$ ) of GRB 081029 by using the two-step energy injection model. The observed data points are taken from Nardini et al (2011). The solid line is our theoretical light curve for GRB 081029 by using the same model as in Figure 1.

Méseáros \& Rees 1997b). When the injected energy is of a kinetic form, not of a Poynting flux, then during the injection process, reverse shocks might be formed. Emission from such reverse shocks could significantly enhance the rebrightenings (Zhang \& Méseáros 2002). In realistic cases, it is also possible that when the fast shell catches up with the slow shell and gives birth to an energy injection, the relative speed between the two colliding shells is not too high, so that only a mildly relativistic reverse shock is generated (Rees \& Méseáros 1998; Kumar \& Piran 2000; (Zhang \& Méseáros 2002)). In this case, emission from the reverse shock will not be very strong. In our modeling, both of the two energy injections last for thousands of seconds. Although the injection flows are assumed to be kinetic-energy-dominated, we believe that the collisions during the injection process would not be too violent and the induced reverse shock would not be too strong. So we have omitted the emission component of the reverse shock. In the future, when more detailed studies are carried out, the effects of the reverse shock should be included.

\section{CONCLUSION AND DISCUSSION}

GRBs are widely believed to be produced by relativistically expanding blastwaves at cosmological dis- 
to the launch of the Swift satellite. Many remarkable and unexpected features such as rebrightenings in the optical afterglows have been found, challenging the view that the optical afterglow light curves should be smooth (Laursen \& Stanek 2003), which is formed since the discovery of the first gamma-ray burst afterglow (Sahu et al. 1997). GRB 081029 is characterized by a complex optical light curve. A distinguishing feature of this event is the obvious rebrightening in the optical band at around $3000 \mathrm{~s}$ after the burst. In this paper, we calculate the overall dynamical evolution of the blastwave numerically by adopting the energy injection model to reproduce the X-ray and optical afterglow light curves of GRB 081029. We assume that the relativistic shock expands in a uniform ISM. We show that the remarkable rebrightening observed in optical band can be satisfactorily modeled by our model. We argue that the rapid rise is due to the energy injection from the late-time interaction of a slow shell with the forward shock. In fact, similar mechanism of energy injection has also been used to explain the afterglows of some other GRBs, such as GRB 010222 (Björnsson et al. 2002), GRB 021004 (Björnsson et al. 2004), GRB 021004 (de Ugarte Postigo et al. 2005), GRB 030329 (Huang et al. 2006) and GRB 051221A (Fan \& Xu 2006). In our calculations, many of the parameters, such as the power-law index of the energy distribution of electrons $(p)$, the electron energy fraction $\left(\epsilon_{e}\right)$, the magnetic energy fraction $\left(\epsilon_{B}\right)$, the initial half opening angle of the ejecta $(\theta)$, have been evaluated typically.

In our model, we have assumed two periods of energy injection, each with a constant injection power. One injection starts at $2.8 \times 10^{3} \mathrm{~s}$ and ends at $t_{\text {end }}=5.3 \times 10^{3} \mathrm{~s}$, with a power of $Q=7.0 \times 10^{47} \mathrm{erg}$ $\mathrm{s}^{-1}$. This energy injection is mainly engaged to account for the rapid rebrightening at about $3000 \mathrm{~s}$. The other injection starts at $8.0 \times 10^{3} \mathrm{~s}$ and ends at $t_{\text {end }}=1.3 \times 10^{4} \mathrm{~s}$, with the power being $Q=3.5 \times 10^{47}$ $\mathrm{erg} \mathrm{s}^{-1}$. This energy injection can help to explain the slight rebrightening at about $10000 \mathrm{~s}$. Physically, this kind of energy injections can be produced by the fallback of materials onto the central compact object of the burster. The fallback is usually continuous, but clumps sometimes could exist in the falling material. When a large clump suddenly plunges into the accretion disk, the accretion rate can be significantly increased, giving birth to a strong outflow. The relativistic shell resulted from the energetic outflow moves outward at approximately a constant speed in a dilute environment that has been swept-up by the previous external shock. It can finally catch up with the fireball material and inject the energy into the fireball, producing a significant rebrightening in the afterglow.

In our fitting to the optical afterglow of GRB 081029, extinction has been taken into account. The theoretical light curve of GRB081029 in the optical band was shifted downward by about 1.57 mag. It is consistent with the result derived by Holland et al. (2012) who suggested that the rest frame $V$ band extinction is $A_{V} \leq 2 \mathrm{mag}$. Extinction has also been considered in many other GRBs. Sokolov et al (2001) pointed out that there is a significant internal extinction in the host galaxies of GRB 970508, GRB 980613, GRB 980703, GRB 990123 and GRB 991208. Rol et al (2007) suggested a high internal extinction, at least 2.3 magnitudes at the infrared $(\mathrm{J})$ wavelength and 5.4 magnitudes at $\mathrm{U}$ Band in the rest-frame to explain the absence of an optical afterglow for GRB 051022, which is a prototypical dark burst. For high redshift GRBs, Draine (2000) draw the conclusion that absorption by vibrationally-excited $H_{2}$ could be responsible for the pronounced drop in flux between $\mathrm{R}$ and I band. Considering the fact that the redshift of GRB 081029 
flux density of GRB 081029 is much less than the theoretical value. Kong et al (2009) derived the extinction of the host galaxy of GRB 980703 as $A_{V} \sim 2.5$ mag by modeling the multi-band afterglow light-curves.

In conclusion, we have shown that our model can reasonably explain both the X-ray and optical afterglow light curves of GRB 081029. Especially the observed optical rebrightening can be fitted quite well by assuming a constant energy injection. In the future, more detailed studies on the energy injection processes will be helpful to provide important clues on the origin and the trigger mechanism of GRBs.

Acknowledgements This work was supported by the National Natural Science Foundation of China (Grant No. 11033002) and the National Basic Research Program of China (973 Program, Grant No. 2009CB824800).

\section{References}

Bersier, D. 2012, arXiv:1206.6979

Björnsson, G., Gudmundsson, E. H., \& Jóhannesson, G. 2004, ApJ, 615, L77

Björnsson, G., Hjorth, J., Pedersen, K., \& Fynbo, J. U. 2002, ApJ, 579, L59

Cummings, J. R., Barthelmy, S. D., Baumgartner, W. H., et al. 2008, GRB Coordinates Network, 8447, 1

Dai, Z. G., Huang, Y. F., \& Lu, T. 1999, ApJ, 520, 634

Dai, Z. G., \& Lu, T. 1998, A\&A, 333, L87

Dai, Z. G., \& Wu, X. F. 2003, ApJ, 591, L21

de Ugarte Postigo, A., Castro-Tirado, A. J., Gorosabel, J., et al. 2005, A\&A, 443, 841

Draine, B. T. 2000, ApJ, 532, 273

Eichler, D., Livio, M., Piran, T., \& Schramm, D. N. 1989, Nature, 340, 126

Fan, Y.-Z., \& Xu, D. 2006, MNRAS, 372, L19

Galama, T. J., Vreeswijk, P. M., van Paradijs, J., et al. 1998, Nature, 395, 670

Gehrels, N., Sarazin, C. L., O’Brien, P. T., et al. 2005, Nature, 437, 851

Gehrels, N., Ramirez-Ruiz, E., \& Fox, D. B. 2009, ARA\&A, 47, 567

Holland, S. T., de Pasquale, M., Mao, J., et al. 2011, American Institute of Physics Conference Series, 1358, 130

Holland, S. T., De Pasquale, M., Mao, J., et al. 2012, ApJ, 745, 41

Huang, Y. F., \& Cheng, K. S. 2003, MNRAS, 341, 263

Huang, Y. F., Cheng, K. S., \& Gao, T. T. 2006, ApJ, 637, 873

Huang, Y. F., Dai, Z. G., \& Lu, T. 1998, A\&A, 336, L69

Huang, Y. F., Dai, Z.-g., \& Lu, T. 1999a, Chinese Physics Letters, 16, 775

Huang, Y. F., Dai, Z. G., \& Lu, T. 1999b, MNRAS, 309, 513

Huang, Y. F., Dai, Z. G., \& Lu, T. 2000a, MNRAS, 316, 943

Huang, Y. F., Gou, L. J., Dai, Z. G., \& Lu, T. 2000b, ApJ, 543, 90

Huang, Y. F., Lu, Y., Wong, A. Y. L., \& Cheng, K. S. 2007, ChJAA (Chin. J. Astron. Astrophys.), 7, 397

Huang, Y. F., Wu, X. F., Dai, Z. G., Ma, H. T., \& Lu, T. 2004, ApJ, 605, 300 
Kong, S. W., Huang, Y. F., Cheng, K. S., \& Lu, T. 2009, Science in China G: Physics and Astronomy, 52, 2047

Kong, S. W., Wong, A. Y. L., Huang, Y. F., \& Cheng, K. S. 2010, MNRAS, 402, 409

Kumar, P., \& Piran, T. 2000, ApJ, 532, 286

Laursen, L. T., \& Stanek, K. Z. 2003, ApJ, 597, L107

Lazzati, D., Rossi, E., Covino, S., Ghisellini, G., \& Malesani, D. 2002, A\&A, 396, L5

Liu, X. W., Wu, X. F., \& Lu, T. 2008, A\&A, 487, 503

MacFadyen, A. I., \& Woosley, S. E. 1999, ApJ, 524, 262

Mészáros, P. 2002, ARA\&A, 40, 137

Meszaros, P., \& Rees, M. J. 1997a, ApJ, 476, 232

Meszaros, P., \& Rees, M. J. 1997b, ApJ, 482, L29

Nakar, E. 2007, Phys. Rep., 442, 166

Narayan, R., Paczynski, B., \& Piran, T. 1992, ApJ, 395, L83

Nardini, M., Greiner, J., Krühler, T., et al. 2011, A\&A, 531, A39

Paczynski, B. 1998, ApJ, 494, L45

Piran, T. 1999, Phys. Rep., 314, 575

Piran, T. 2000, Phys. Rep., 333, 529

Rees, M. J., \& Meszaros, P. 1994, ApJ, 430, L93

Rees, M. J., \& Meszaros, P. 1998, ApJ, 496, L1

Rhoads, J. E. 1999, ApJ, 525, 737

Rol, E., van der Horst, A., Wiersema, K., et al. 2007, ApJ, 669, 1098

Sahu, K. C., Livio, M., Petro, L., et al. 1997, Nature, 387, 476

Sakamoto, T., Baumgartner, W. H., Burrows, D. N., et al. 2008, GRB Coordinates Network, 8435, 1

Sari, R., \& Esin, A. A. 2001, ApJ, 548, 787

Sari, R., Piran, T., \& Halpern, J. P. 1999, ApJ, 519, L17

Sokolov, V. V., Fatkhullin, T. A., Castro-Tirado, A. J., et al. 2001, A\&A, 372, 438

Sokolov, V. V., Zharikov, S. V., Baryshev, Y. V., et al. 1999, A\&A, 344, 43

Tam, P. H., Pun, C. S. J., Huang, Y. F., \& Cheng, K. S. 2005, New Astronomy, 10, 535

Usov, V. V. 1994, MNRAS, 267, 1035

van Eerten, H. J., Leventis, K., Meliani, Z., Wijers, R. A. M. J., \& Keppens, R. 2010, MNRAS, 403, 300

Wei, D. M., \& Lu, T. 2000, A\&A, 360, L13

Woosley, S. E. 1993, ApJ, 405, 273

Woźniak, P. R., Vestrand, W. T., Wren, J. A., et al. 2006, ApJ, 642, L99

Zhang, B. 2007, ChJAA (Chin. J. Astron. Astrophys.), 7, 1

Zhang, B., \& Mészáros, P. 2001a, ApJ, 552, L35

Zhang, B., \& Mészáros, P. 2001b, ApJ, 559, 110

Zhang, B., \& Mészáros, P. 2002, ApJ, 566, 712 